\title{
Correction to: manuscript entitled: MO-NFSA for solving unconstrained multi-objective optimization problems
}

\author{
Weng-Hooi $\operatorname{Tan}^{1} \cdot$ Junita Mohamad-Saleh ${ }^{1}$ (])
}

Published online: 25 February 2021

(c) Springer-Verlag London Ltd., part of Springer Nature 2021

\section{Correction to: Engineering with Computers https://doi.org/10.1007/s00366-020-01223-4}

Below are the Corrections Given for Proof-Read:

1) Requested correction to: Add in missing Fig. 6c

Based on the corrections we requested in the proofreading, we only asked for corrections to Fig. $6 a, b$ due to their poor qualities, but there was no comment for deleting or removing Fig. 6c. However, in the in-Press version, Fig. 6c was removed from the article. Figure $6 \mathrm{c}$ is attached below for inclusion.

2) Requested correction to: To add missing part of a sentence

In the last sentence of Sect. 4.1 on page 7 of the e-offprint, the correction to ass missing point in the sentence was not made based on our comments in the proofreading. The additional part of the last sentence must be added at the end of the last sentence in Sect. 4.1.

(Wrong) Text content in published manuscript:

where Eq. (14) contains the elements of normative communication behavior and normative memory behavior in an expression, $\boldsymbol{X}_{i}^{t}$ represents the feasible solution of $i$ th candidate at $t$ th iteration, $\boldsymbol{X}_{\text {gbest }}^{t}$ indicates the leader solution selected at $t$ th iteration, $\boldsymbol{X}_{\text {gbest }}^{t-1}$ indicates the leader solution selected at $(t-1)$ th iteration, $\boldsymbol{X}_{i, l b e s t}^{t}$ denotes the locally stored solution of $i$ th candidate at $t$ th iteration, and $\boldsymbol{X}_{i, l b e s t}^{t-1}$ denotes the locally stored solution of $i$ th candidate at $(t-1)$ th iteration. $\boldsymbol{X}_{c g}^{t}$ and $\boldsymbol{X}_{c g}^{t-1}$ can be obtained using Eqs. (4) and

The original article can be found online at https://doi.org/10.1007/ s00366-020-01223-4.

Junita Mohamad-Saleh

jms@usm.my

Weng-Hooi Tan

tanwenghooi@hotmail.com

1 School of Electrical and Electronic Engineering, Universiti Sains Malaysia, Nibong Tebal, 11430 Penang, Malaysia
(5), respectively, while $\boldsymbol{X}_{i, c l}^{t}$ and $\boldsymbol{X}_{i, c l}^{t-1}$ can be obtained using Eqs. (8) and (9), respectively.

(Correct) Text content as per requested:

where Eq. (14) contains the elements of normative communication behavior and normative memory behavior in an expression, $\boldsymbol{X}_{i}^{t}$ represents the feasible solution of $i$ th candidate at $t$ th iteration, $\boldsymbol{X}_{\text {gbest }}^{t}$ indicates the leader solution selected at $t$ th iteration, $\boldsymbol{X}_{\text {gbest }}^{t-1}$ indicates the leader solution selected at $(t-1)$ th iteration, $\boldsymbol{X}_{i, l b e s t}^{t}$ denotes the locally stored solution of $i$ th candidate at $t$ th iteration, and $\boldsymbol{X}_{i, l \text { lbest }}^{t-1}$ denotes the locally stored solution of $i$ th candidate at $(t-1)$ th iteration. $\boldsymbol{X}_{c g}^{t}$ and $\boldsymbol{X}_{c g}^{t-1}$ can be obtained using Eqs. (4) and (5), respectively, while $\boldsymbol{X}_{i, c l}^{t}$ and $\boldsymbol{X}_{i, c l}^{t-1}$ can be obtained using Eqs. (8) and (9), respectively, but the concept of greedy selection in these equations must be replaced with the concept of dominance.

3) Requested correction to: Delete unnecessary word in a paragraph

On line 20 of Sect. 3.1 on page 4 of e-offprint, we requested that the word "AF" be deleted from the sentence, but it still remains in the e-offprint version.

(Wrong) Text content in published manuscript:

where $\boldsymbol{X}_{\text {gbest }}^{t}$ indicates the global best location vector of the AF population at $t$ th iteration, $\boldsymbol{X}_{\text {gbest }}^{t-1}$ indicates the global best location vector of the population at $(t-1)$ th iteration, $\boldsymbol{X}_{c g}^{t}$ represents the guidelines selected within the reconnaissance range which originate from $\boldsymbol{X}_{g b e s t}^{t}$, and $\boldsymbol{X}_{c g}^{t-1}$ represents the guidelines selected within the reconnaissance range which originate from $\boldsymbol{X}_{\text {gbest }}^{t-1}$.

(Correct) Text content as per requested:

where $\boldsymbol{X}_{\text {gbest }}^{t}$ indicates the global best location vector of the population at $t$ th iteration, $\boldsymbol{X}_{\text {gbest }}^{t-1}$ indicates the global best location vector of the population at $(t-1)$ th iteration, $\boldsymbol{X}_{c g}^{t}$ represents the guidelines selected within the reconnaissance range which originate from $\boldsymbol{X}_{g b e s t}^{t}$, and $\boldsymbol{X}_{c g}^{t-1}$ represents the guidelines selected within the reconnaissance range which originate from $\boldsymbol{X}_{\text {gbest }}^{\boldsymbol{t}}$.

4) Requested correction to: Add in missing equation in Table 3 


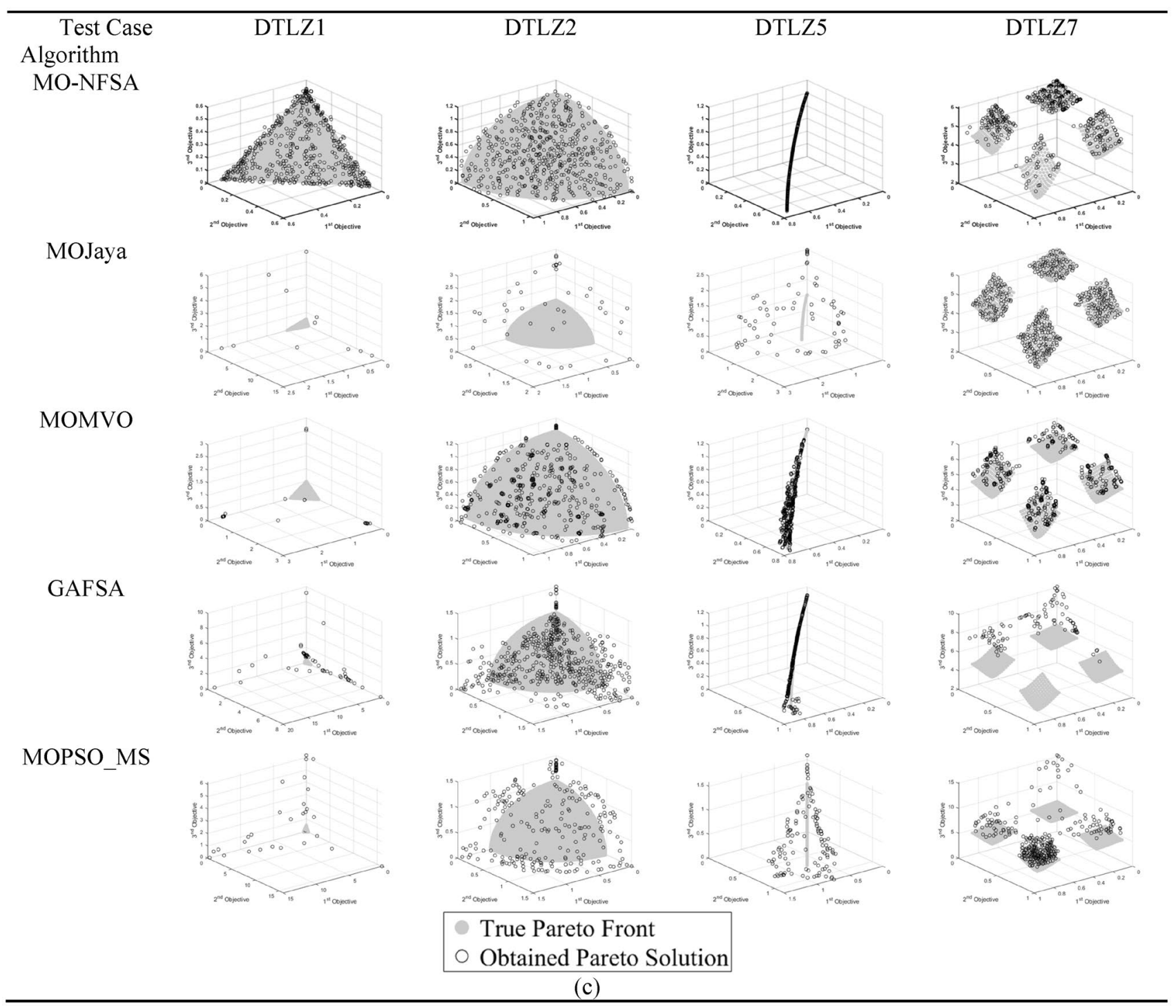

Fig. 6 Pareto front obtained by each algorithm in a fixed dimensional multi-objective optimization test cases, b non-fixed dimensional multiobjective optimization test cases, and $\mathbf{c}$ scalable multi-objective optimization test cases

There is an equation missing from Table 3. Please update the entire table by using the following replacement table to avoid further missing equations. where $\left|x_{M}\right|=k=N-M+1 . x_{M}$ is the last $k$ variables of $X$, which can be defined as $X_{M \sim N}$.

The original article has been corrected. 
Table 3 Scalable multi-objective optimization test cases

\begin{tabular}{|c|c|c|c|c|}
\hline Test Case & Mathematical Expression & {$[L, U]$} & $N$ & Remarks \\
\hline DTLZ1 & $\begin{array}{l}f_{1}(X)=\frac{1}{2} X_{1} X_{2} \ldots X_{M-1}\left(1+g\left(x_{M}\right)\right) \\
f_{2}(X)=\frac{1}{2} X_{1} X_{2} \ldots\left(1-X_{M-1}\right)\left(1+g\left(x_{M}\right)\right) \\
\qquad \begin{array}{c}\vdots \\
f_{M-1}(X)=\frac{1}{2} X_{1}\left(1-X_{2}\right)\left(1+g\left(x_{M}\right)\right)\end{array} \\
\qquad f_{M}(X)=\frac{1}{2}\left(1-X_{1}\right)\left(1+g\left(x_{M}\right)\right) \\
\text { where: } \\
g\left(x_{M}\right)=100\left[\left|x_{M}\right|+\sum_{X_{k} \in x_{M}}\left(X_{k}-0.5\right)^{2}-\cos \left(20 \pi\left(X_{k}-0.5\right)\right)\right]\end{array}$ & {$[0,1]$} & 5 & Linear \\
\hline DTLZ2 & $\begin{array}{l}f_{1}(X)=\left(1+g\left(x_{M}\right)\right) \cos \left(\frac{X_{1} \pi}{2}\right) \ldots \cos \left(\frac{X_{M-2} \pi}{2}\right) \cos \left(\frac{X_{M-1} \pi}{2}\right) \\
f_{2}(X)=\left(1+g\left(x_{M}\right)\right) \cos \left(\frac{X_{1} \pi}{2}\right) \ldots \cos \left(\frac{X_{M-2} \pi}{2}\right) \sin \left(\frac{X_{M-1} \pi}{2}\right) \\
\qquad f_{3}(X)=\left(1+g\left(x_{M}\right)\right) \cos \left(\frac{X_{1} \pi}{2}\right) \ldots \sin \left(\frac{X_{M-2} \pi}{2}\right) \\
\qquad \quad \vdots \\
\text { where: } \\
g\left(x_{M}\right)=\sum_{X_{k} \in x_{M}}(X)=\left(1+g\left(X_{M}\right)\right) \sin \left(\frac{X_{1} \pi}{2}\right)\end{array}$ & {$[0,1]$} & 30 & Concave \\
\hline DTLZ3 & $\begin{array}{l}f_{1}(X)=\left(1+g\left(x_{M}\right)\right) \cos \left(\frac{X_{1} \pi}{2}\right) \ldots \cos \left(\frac{X_{M-2} \pi}{2}\right) \cos \left(\frac{X_{M-1} \pi}{2}\right) \\
f_{2}(X)=\left(1+g\left(x_{M}\right)\right) \cos \left(\frac{X_{1} \pi}{2}\right) \ldots \cos \left(\frac{X_{M-2} \pi}{2}\right) \sin \left(\frac{X_{M-1} \pi}{2}\right) \\
\qquad f_{3}(X)=\left(1+g\left(x_{M}\right)\right) \cos \left(\frac{X_{1} \pi}{2}\right) \ldots \sin \left(\frac{X_{M-2} \pi}{2}\right) \\
\qquad f_{M}(X)=\left(1+g\left(x_{M}\right)\right) \sin \left(\frac{X_{1} \pi}{2}\right) \\
\text { where: } \\
g\left(x_{M}\right)=100\left[\left|x_{M}\right|+\sum_{X_{k} \in x_{M}}\left(X_{k}-0.5\right)^{2}-\cos \left(20 \pi\left(X_{k}-0.5\right)\right)\right]\end{array}$ & {$[0,1]$} & 5 & Concave \\
\hline DTLZ4 & 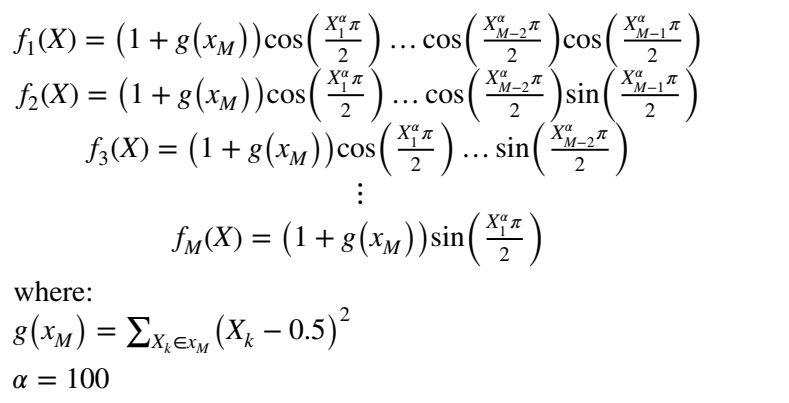 & {$[0,1]$} & 30 & Concave \\
\hline DTLZ5 & $\begin{array}{l}f_{1}(X)=\left(1+g\left(x_{M}\right)\right) \cos \left(\frac{X_{1} \pi}{2}\right) \ldots \cos \left(\frac{\theta_{M-2} \pi}{2}\right) \cos \left(\frac{\theta_{M-1} \pi}{2}\right) \\
f_{2}(X)=\left(1+g\left(x_{M}\right)\right) \cos \left(\frac{X_{1} \pi}{2}\right) \ldots \cos \left(\frac{\theta_{M-2} \pi}{2}\right) \sin \left(\frac{\theta_{M-1} \pi}{2}\right) \\
\qquad f_{3}(X)=\left(1+g\left(x_{M}\right)\right) \cos \left(\frac{X_{1} \pi}{2}\right) \ldots \sin \left(\frac{\theta_{M-2} \pi}{2}\right) \\
\qquad f_{M}(X)=\left(1+g\left(x_{M}\right)\right) \sin \left(\frac{X_{1} \pi}{2}\right) \\
\text { where: } \quad \pi \\
\theta_{k}=\frac{\pi}{4\left(1+g\left(x_{M}\right)\right)}\left(1+2 g\left(x_{M}\right) X_{k}\right), k=2,3, \ldots,(M-1) \\
g\left(x_{M}\right)=\sum_{X_{k} \in x_{M}}\left(X_{k}-0.5\right)^{2}\end{array}$ & {$[0,1]$} & 30 & Continuous curve \\
\hline
\end{tabular}


Table 3 (continued)

\begin{tabular}{|c|c|c|c|c|}
\hline Test Case & Mathematical Expression & {$[L, U]$} & $N$ & Remarks \\
\hline DTLZ6 & 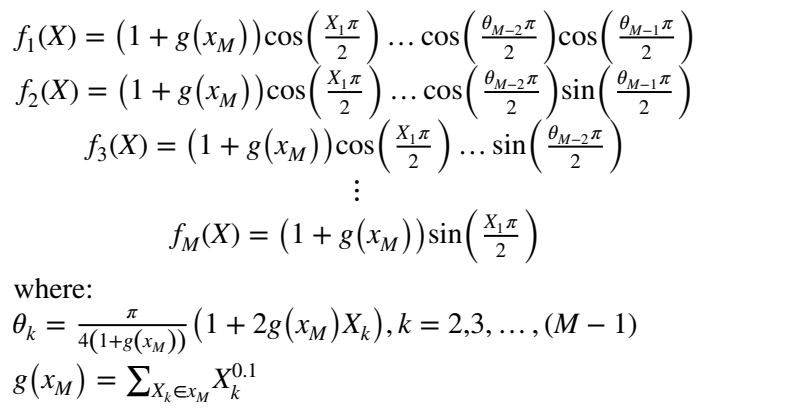 & {$[0,1]$} & 5 & Continuous curve \\
\hline DTLZ7 & $\begin{array}{l}\qquad \begin{array}{l}f_{1}(X)=X_{1} \\
f_{2}(X)=X_{2} \\
\vdots\end{array} \\
f_{M-1}(X)=X_{M-1} \\
f_{M}(X)=\left(1+g\left(x_{M}\right)\right) h\left(f_{1}, f_{2}, \ldots, f_{M-1}, g\left(x_{M}\right)\right) \\
\text { where: } \\
g\left(x_{M}\right)=1+\frac{9}{\left|x_{M}\right|} \sum_{X_{k} \in x_{M}} X_{k} \\
h\left(f_{1}, f_{2}, \ldots, f_{M-1}, g\left(x_{M}\right)\right)=M-\sum_{m=1}^{M-1}\left[\frac{f_{m}}{1+g\left(x_{M}\right)}\left(1+\sin \left(3 \pi f_{m}\right)\right)\right]\end{array}$ & {$[0,1]$} & 30 & Discontinuous \\
\hline
\end{tabular}

